\title{
sciendo
}

\section{The Use of Small-Sided Games as an Aerobic Fitness Assessment Supplement within Elite Level Professional Soccer}

\author{
by \\ Adam Lee Owen ${ }^{1}$, Matthew Newton ${ }^{2}$, Aidan Shovlin ${ }^{3}$, Shane Malone ${ }^{3}$
}

The purpose of this investigation was to quantify the association between 5 vs. 5 small sided games (SSG) running performance and physiological performance during the Yo-YoIR1 test to ascertain the utility of SSGs as a potential fitness test modality within elite professional soccer players. Twenty-three $(n=23)$ elite male professional soccer players (mean $\pm S D$ age $25.3 \pm 3.1$ yrs, mass: $76 \pm 9 \mathrm{~kg}$, height: $176 \pm 9 \mathrm{~cm}$ ) were assessed. Players completed an intermittent aerobic fitness test (Yo-YoIR1) and a 5 vs. 5 SSGs protocol for the purpose of the study. During all SSGs players wore GPS (Statsports 10-Hz, Viper Pod, Newry, Northern Ireland) and HR monitors (Polar, Oy Kemple, Finland) with these measures related to Yo-YoIR1 running performance. Results revealed SSGs running performance $(\mathrm{TD} ; \mathrm{m})$ and physiological performance $(H R)$ showed the lowest $C V \%(<5 \%)$, with high speed movements, accelerations and decelerations highlighting higher CV\% during SSGs. Possibly small to possibly very large associations were observed for running performance during 5 vs. 5 SSGs and Yo-YoIR1 performance, with negative associations observed between physiological performance during SSG and YoYoIR1 running performance. To conclude, the current study observed how running performance during a standardised 5 vs. 5 SSG protocol within elite soccer cohorts is associated with the Yo-YoIR1 running performance. Given the low CV\%, repeatability and large association of global running performance and internal load measures during a 5 vs. 5 SSG with Yo-YoIR1 performance, this particular soccer specific SSG protocol potentially supplements traditional non-sport specific testing assessments.

Key words: soccer, training, testing, fitness, assessment, GPS.

\section{Introduction}

The demands of soccer match-play have been reported (Casamichana et al., 2012; Malone et al., 2016; Taylor et al., 2018). During official games players complete between 9 and $14 \mathrm{~km}$ (Manzi et al., 2014; Osgnach et al., 2010), with a high proportion of distance covered jogging and walking (Manzi et al., 2014). However, the outcome of a soccer match is heavily influenced by the high-speed components of play despite the game primarily taxing the aerobic system (Manzi et al., 2014). Notably, $\sim 300$ acceleration and deceleration efforts (when categorized as changes in movement above $0.5 \mathrm{~m} \cdot \mathrm{s}-2$ ) are performed per half (30) and $~ 18 \%$ of the total distance covered during a soccer match is completed while accelerating or decelerating (Akenhead et al., 2016; Manzi et al., 2014). Given the need for coaches to train technical, tactical and physiological stimuli during training, the majority of soccer-based training is completed through the use of small-sided games (SSGs). These games represent a concomitant form of training allowing for many physical qualities to be trained within shortened pitch dimensions across specific player numbers and game formats that also allow players to engage in sport-specific decision

\footnotetext{
1 - Inter-university Laboratory of Human Movement Biology(EA 7424), Claude Bernard Lyon 1, Lyon, France.

2 - The Tom Reilly Building, Research Institute for Sport and Exercise Sciences, Liverpool John Moores University, Liverpool, UK.

3 - Human Performance Lab, Department of Science, Technological University Dublin, Tallaght, Dublin, Ireland.
} 
making (Fleay et al., 2018; Owen et al., 2012).

Indeed, within many team sports SSG are widely utilised by coaches to develop aerobic and anaerobic components of fitness within players, with these games shown to provide similar physiological stimuli as interval training (Delall et al., 2012). However, when organizing SSGs, coaches who want to achieve physiological and physical performances that allow for the development of aerobic endurance need to consider a number of different factors which may affect exercise intensity. These factors include the number of players and the pitch size (Djaoui 2017; Owen et al., 2014), the game rules (Halouani et al., 2014; Malone et al., 2019), coach encouragement (Halouani et al., 2014; Kolu et al., 2015; Malone et al., 2017), the absence or presence of goalkeepers (Kolu et al., 2015), and training regime (Mallo et al., 2008). Additionally, bout duration (Kolu et al., 2015), goal size (Malone et al., 2019) and work to rest ratios (Malone et al., 2019) should be considered. Furthermore, the variability of SSGs with a standardised format has been found to be low when assessing both the heart rate and total distance (coefficient of variation $(\mathrm{CV})<5 \%$ ) (HillHaas et al., 2011; Mallo et al., 2008; Owen et al., 2014). This is despite larger variability being reported in higher-speed distances (Hill-Haas et al., 2011; Mallo et al., 2008; Rampinini et al., 2007). As a result of the recent findings surrounding SSGs, it may be suggested that through the use of smaller standardised training games, coaches have the possibility to utilise these games to detect worthwhile changes in running and physiological performance that may be linked to increased fitness capacities. Unlike match play SSG performance if standardised, is less position, score-line and opposition dependant. Therefore, the physical and physiological performance during these games could potentially be used to highlight aerobic fitness changes within players, removing the need for maximal fitness assessments such as the time-trials, linear maximal running testing and shuttle testing. Previously Stevens et al. (2016) have shown that a 6 vs. 6 SSG showed moderate to large correlation with YoYoIR2 performance for total distance $(\mathrm{r}=$ 0.45; 90\%CI: 0.31-0.56; moderate), high speed distance ( $\mathrm{r}=0.70 ; 90 \% \mathrm{CI}: 0.61-0.77$; large) and high accelerations $(\mathrm{r}=0.59$; 90\%CI: $0.48-0.68$; large) showing that SSG performance can relate to testing performance for soccer cohorts. However, the above study placed caution with regard to the application of SSGs as fitness tests due to these games having poor validity measures within the tested cohort.

In order to best ascertain sport specific training adaptations, coaches will engage in specific testing batteries at given time points during the competitive season, however, no such soccer specific assessments have been reported which allow technical and physical staff to test within training situations in order to maximise training content. Several test protocols are used and well-reported within the literature to assess specific physical qualities, such as maximal (repeated) sprinting and aerobic and anaerobic endurance tests, e.g. the Yo-Yo tests, however, none are fully integrated with a sport-specific technical involvement. Furthermore, recent analysis by Malone et al. (2018) has shown that improved intermittent aerobic fitness capacities result in lower odds risk of injury within soccer cohorts, additionally improved aerobic capacity increases coaches' ability to increase the amount of the prescribed training load, high speed and sprint running across training cycles. However, most of these intermittent aerobic testing batteries require players to complete maximal efforts over specific time duration depending on the test selected. Due to the competitive schedule within elite soccer and periods of fixture congestion these maximal testing protocols may not be feasible for coaches to run due to tim constraints and increased levels of fatigue amongst players (Halson, 2014). Given that standardised SSGs have shown a low CV for physical and physiological variables and that SSGs are a regular component within elite soccer training, the aim of the current investigation was to determine whether a 5 vs. 5 SSG protocol could be utilised as a surrogate of aerobic fitness within an elite soccer cohort. To determine it, we first sought to understand the validity and reliability of the 5 vs. 5 SSG and secondly, sought to understand the association between running performance measures within SSGs and YoYoIR1 performance. As a result of the unique focus of this current investigation, the potential to develop and propose a soccer specific assessment protocol amongst elite level professional soccer players as a supplement to the traditional current methods 
already well-documented, led to the development of the research topic. It was hypothesised that the 5 vs. 5 SSG would have limited variation for total distance and HR measures and that there would be a strong positive association between the SSG protocol performance and Yo-YoIR1 performance.

\section{Methods}

\section{Participants}

The current investigation was an observation study of elite level professional soccer players competing for a team that at the time of data collection were competing within the top tier of their respective league competition. Additionally, $80 \%$ of the players assessed were representing their respective European national teams at the time of investigation. Data were collected for 23 players (Mean $\pm S D$, age: $25.3 \pm 3.1$ years; body height: $183 \pm 7 \mathrm{~cm}$; body mass: $72 \pm 7$ $\mathrm{kg}$ ) over one season. The study was approved by the local institute's research ethics committee (Technological University Dublin, Tallaght, Ireland) and written informed consent was obtained from each participant. The study period involved all training sessions during the 2017/2018 season.

\section{Small-Sided Game (5 vs. 5)}

During the investigation period, a total of 1560 individual SSG data points and metrics were assessed with a median of 28 observations per player. The SSG consisted of free play with the focus of the SSG to keep possession in a 5 vs. 5 method within a $25 \times 25 \mathrm{~m}$ grid resulting in a relative player area of $62.5 \mathrm{~m}^{2}$. All SSGs were performed with a continuous rhythm, under the supervision and motivation of several coaches in order to keep running performance of players high (Manzi et al., 2014). During all SSGs free play was allowed with maximal touches, in all cases multiple replacement balls were available for prompt replacement when hit out of play (Djaoui et al., 2017). Before the study period, these SSGs were frequently performed to ensure familiarisation before the experimental period. All sessions were performed on the same natural grass pitch. In addition, all exercise games were performed at the same time of the day (10:30 11:00 am) to limit to effects of circadian variations on measured variables (Kolu et al., 2015). All of the SSG assessment protocols were completed after a standardised warm up of $15 \mathrm{~min}$. All games were standardised for time $(3 \mathrm{~min}$ in length) with players performing 3 repetitions of 3 min games within a single session and a $2 \mathrm{~min}$ passive recovery period between repetitions of the protocol. All games were coach driven through verbal encouragement to ensure maximum effort.

\section{Yo-Yo Intermittent Recovery Test Level 1}

The Yo-YoIR1 consists of $2 \times 20 \mathrm{~m}$ shuttle runs performed at increasing speeds, with $10 \mathrm{~s}$ of active recovery between runs (intermittent recovery version). The Yo-YoIR1 has been reported to assess a player's endurance capacity with a high aerobic energy contribution. During the current investigation testing was completed in line with the procedures described in a previous study (Malone et al., 2017). This included participants completing a 15-min dynamic warmup involving multi-joint and running activities of progressive intensity. Failure to complete a shuttle resulted in a verbal warning with participants being withdrawn on a second failure. Total distance and corresponding maximum speed at the final completed shuttle were recorded at the end of the test. The heart rate was measured at $1 \mathrm{~s}$ intervals during the test using the Polar Team 2 System (Polar Electro Oy, Kemple, Finland). Stored data were then exported out of the dedicated Polar software (Polar Team 2 Software, Kemple, Finland) to a bespoke data base (Microsoft Excel, Redmond, USA). The YoYoIR1 test has been previously shown valid and reliable with the distance covered during a Yo-YoIR1 being directly related to match play running performance within soccer in addition to being sensitive to changes in fitness across acute training periods in team sports (Lacome et al., 2017; Malone et al., 2017).

\section{Running Performance Monitoring}

During SSGs players' running performance was monitored using a portable nondifferential $10-\mathrm{Hz}$ GPS device integrated with a $100 \mathrm{~Hz}$, 3-dimensional accelerometer, 3dimensional gyroscope, and a 3-dimensional digital compass (STATSports, 10-Hz, Viper Pod, Northern Ireland). This type of a system has previously been shown to provide valid and reliable estimates of instantaneous velocity during acceleration, deceleration, and constant-velocity movements during linear, multidirectional, and soccer-specific activities (Beato et al., 2016). Each player was assigned a GPS vest that was tightly 
fitted to their upper torso, holding the receiver between the scapulae. All devices were always activated 15 minutes before the data collection to allow acquisition of satellite signals in accordance with the manufacturer's instructions. In addition, to avoid inter-unit error, each player wore the same GPS device for each training session (Malone et al., 2016) After recording, the data were downloaded to a computer and analysed using the software package Viper version (STATSports, Viper, System). Based on GPS data, total, high-speed $\left(>19.8 \mathrm{~km} \cdot \mathrm{h}^{-1}\right)$ and sprint distance $\left(>25.5 \mathrm{~km} \cdot \mathrm{h}^{-1}\right)$, as well as average metabolic power $\left(\mathrm{W} \cdot \mathrm{kg}^{-1}\right)$, high metabolic power distance $\left(\mathrm{m} ; \geq 20 \mathrm{~W} \cdot \mathrm{kg}^{-1}\right)$, accelerations $(\mathrm{n} ; \geq 3.3$ $\left.\mathrm{m} \cdot \mathrm{s}^{-2}\right)$, decelerations $\left(\mathrm{n} ; \geq-3.3 \mathrm{~m} \cdot \mathrm{s}^{-2}\right)$ and the dynamic stress load (AU) were calculated during each training session. The dynamic stress load represents the weighted total of all accelerometery above $2 \mathrm{G}$ based on convex curved G-force ratings. These measures are reflective of standardised measures of training loads regularly reported within soccer cohorts (Malone et al., 2017; Russell et al., 2016).

\section{Statistical Analysis}

Pearson's correlation $(r)$ was used to assess the association between Yo-YoIR1 distance and selected 5 vs. 5-SSG variables. Magnitude of the correlation $(r)$ was considered trivial $(<0.1)$, small $(>0.1-0.3)$, moderate $(>0.3-0.5)$, large $(>0.5-$ $0.7)$, very large $(>0.7-0.9)$, nearly perfect $(>0.9-$ 1.0), and perfect (1.0) (Hopkins, 2011). Analyses were performed using IBM SPSS Statistics for Windows (Version 22.0). The intraclass correlation coefficient (ICC), typical error (TE) and TE as a coefficient of variation (CV) of selected 5 vs. 5-SSG physical and physiological variables were calculated within a specific spreadsheet for the assessment of ICC created by Hopkins (2000). The reproducibility of 5 vs. 5 SSGs was determined using Bland and Altman (1995) limits of agreement. Reference lines were determined as the mean difference \pm 1.96 standard deviations. All analyses were conducted with the statistical significance set at $p<.05$.

\section{Results}

\section{Validity and Reliability of 5 vs. 5 SSGs}

The ICC $(90 \% \mathrm{CI})$, bias \pm random error and $\mathrm{CV} \%(90 \% \mathrm{CI})$ for running performance measures during SSGs are shown in Table 1.
During these 5 vs. 5 SSGs total distance $(0.94 ; 0.76-$ $0.98)$, the dynamic stress load $(0.94 ; 0.76-0.98)$, average metabolic power $(0.82 ; 0.76-0.91)$, time above $85 \%$ of the $\mathrm{HR}_{\max }(0.86 ; 0.65-0.91)$ and the percentage of the HRmax $(0.87 ; 0.53-0.94)$ showed very large ICCs. Global running performance measures showed the lowest $\mathrm{CV} \%(<5 \%)$ during SSGs. Objective internal load measures (Time above $85 \%$ HRmax, average $H_{R_{\max }} \mathrm{HR}_{\max }$, percentage $\mathrm{HR}_{\max }$ ) also showed low $\mathrm{CV} \%$. Higher speed movement as well as acceleration and deceleration measures presented higher $\mathrm{CV} \%$.

\section{Association between running and physiological measures in SSG and Aerobic Fitness}

Table 2 shows typical running and physiological performance measures during 5 vs. 5 SSGs within elite soccer players. Table 3 shows the observed association between running measures and explained variance during SSG and Yo-YoIR1 performance measures. Possibly small to possibly very large associations were observed for running performance during Yo-YoIR1 and 5 vs. 5 SSG running performance. Total distance (0.88; 0.67-0.91; possibly very large) during SSGs had a possibly very large association with YoYoIR1 running performance in addition to the dynamic stress load $(0.80 ; 0.67-0.91$; possibly very large) that showed similar possibly very large associations. Likely large associations were observed between high speed running and average metabolic power during 5 vs. 5 SSG and Yo-YoIR1 running performance, with negative associations observed between objective internal measures during SSG and YoYoIR1 running performance.

\section{Discussion}

The current investigation sought to explore the possibility of utilising SSGs as an assessment of aerobic fitness within elite soccer players. We firstly assessed the reliability and repeatability of these SSGs, with the main findings highlighting the lowest CV\% (<5\%) showed by global running performance measures (total distance, average metabolic power, dynamic stress load) during SSGs. Furthermore, physiological measures (time above $85 \% \mathrm{HR}_{\max }$, average $H_{\text {max }}, H_{\max }$, percentage $H_{\text {max }}$ ) also showed low CV\% during 5 vs. 5 SSGs. When the association between SSG and Yo-YoIR1 running performance was considered, the movement 
metrics recorded by athletes within these SSGs ( 5 vs. 5) were related to the aerobic fitness profile of players. These data may suggest that the standardised SSG protocol employed within the study could potentially be utilised by coaches as a fitness assessment tool within the soccer ergonomic process. Interestingly, total distance $(\mathrm{m})$ and the dynamic stress load (AU) recorded the highest associations with Yo-YoIR1 performance with most movement metrics recording moderate to large associations with YoYoIR1 performance. Based on these findings, it may be suggested that running performance within the standardised SSG protocol may be utilised to assess aerobic fitness within elite soccer cohorts.

Table 1

The typical running and physiological performance measures during 5 vs. 5 SSGs within elite soccer players. Data presented as Mean \pm SD $(90 \%$ CI).

\begin{tabular}{ll}
\hline Yo-YoIR1 (m) & $1349 \pm 167(1100-1578)$ \\
Total Distance (m) & $964 \pm 19(875-1100)$ \\
High Speed Distance (m) & $29 \pm 13(8-47)$ \\
Average Metabolic Load $\left(\mathrm{W} \cdot \mathrm{kg}^{-1}\right)$ & $10 \pm 0.7(9.8-11.8)$ \\
High Metabolic Load Distance $\left(\mathrm{m} ; \geq 20 \mathrm{~W} \cdot \mathrm{kg}^{-1}\right)$ & $199 \pm 45(123-223)$ \\
Sprint Distance (m) & $19 \pm 10(2-33)$ \\
Dynamic Stress Load (AU) & $59 \pm 6(45-68)$ \\
Accelerations (n) & $16 \pm 9(7-21)$ \\
Decelerations (n) & $18 \pm 7(8-33)$ \\
Time Above 85\% HRmax $(\%)$ & $4.3 \pm 0.8(3.1-5.1)$ \\
Average HR (BPM; HRavg) & $167 \pm 17(150-179)$ \\
Maximal HR (BPM; HRmax) & $179 \pm 23(167-198)$ \\
Percentage HRmax $\%$ ) & $87 \pm 6(81-97)$ \\
RPE (AU; CR10) & $6.3 \pm 1.4(4.1-7.3)$ \\
\hline
\end{tabular}

Table 2

The Intraclass correlation coefficient (ICC; $90 \%$ CI), bias \pm random error calculated through Bland and Altman analysis, coefficient of variation (CV; $90 \% \mathrm{CI}$ ) of a 5 vs. 5 side small-sided game for GPS and physiological measures.

\begin{tabular}{lccc}
\hline SSG Measures & ICC $\%(90 \% \mathrm{CI})$ & Bias \pm Random Error & CV\% (90\% CI) \\
\hline Total Distance (m) & $0.94(0.76-0.98)$ & $0.8 \pm 5.4$ & $2.5(1.8-3.9)$ \\
High Speed Distance (m) & $0.65(0.45-0.81)$ & $0.1 \pm 1.2$ & $8.1(2.1-10.1)$ \\
Average Metabolic Load (W·kg-1) & $0.82(0.76-0.91)$ & $1.3 \pm 7.5$ & $3.4(1.1-5.1)$ \\
High Metabolic Load Distance (m) & $0.78(0.69-0.81)$ & $0.8 \pm 1.9$ & $6.1(4.1-10.0)$ \\
Sprint Distance (m) & $0.77(0.66-0.85)$ & $0.1 \pm 2.9$ & $16.1(10.1-20.2)$ \\
Dynamic Stress Load (AU) & $0.94(0.76-0.98)$ & $0.8 \pm 5.4$ & $2.5(1.8-3.9)$ \\
Accelerations (n) & $0.61(0.55-0.73)$ & $0.3 \pm 2.5$ & $14.1(8.1-23.1)$ \\
Decelerations (n) & $0.67(0.51-0.71)$ & $0.1 \pm 2.9$ & $16.2(9.1-21.3)$ \\
Time Above 85\% HRmax $(\%)$ & $0.85(0.65-0.91)$ & $0.6 \pm 5.4$ & $2.2(1.9-2.7)$ \\
Average HR (Bpm; HRavg) & $0.77(0.67-0.91)$ & $1.5 \pm 10.4$ & $3.0(1.9-4.3)$ \\
Maximal HR (BPM; HRmax) & $0.79(0.71-0.81)$ & $0.3 \pm 4.9$ & $2.0(1.1-3.1)$ \\
Percentage HRmax (\%) & $0.87(0.53-0.94)$ & $0.2 \pm 6.8$ & $2.2(1.1-4.1)$ \\
RPE (AU; CR10) & $0.57(0.23-0.78)$ & $0.1 \pm 1.2$ & $5.5(2.1-6.7)$ \\
\hline
\end{tabular}




\begin{tabular}{|c|c|c|c|}
\hline \multicolumn{4}{|c|}{$\begin{array}{l}\text { Table } 3 \\
\text { The Pearson's correlation coefficients }(r ;(90 \% C I) \text {, magnitude of correlation } \\
\text { and explained variance }\left(R^{2}\right) \text { for GPS and physiological variables during } \\
\text { a } 5 \text { vs. } 5 \text { side small-sided game with Yo-YoIR1 distance for elite soccer players. }\end{array}$} \\
\hline SSG Measures & $r(90 \% \mathrm{CI})$ & $\begin{array}{c}\text { Magnitude of } \\
\text { Correlation }\end{array}$ & $\begin{array}{c}\% \text { of Explained } \\
\text { Variance }\left(\mathrm{R}^{2}\right)\end{array}$ \\
\hline Total Distance (m) & $0.88(0.67$ to 0.91$)$ & possibly very large & $77 \%$ \\
\hline High Speed Distance (m) & $0.66(0.45$ to 0.71$)$ & likely large & $44 \%$ \\
\hline Average Metabolic Load $\left(\mathrm{W} \cdot \mathrm{kg}^{-1}\right)$ & $0.76(0.35$ to 0.89$)$ & likely large & $31 \%$ \\
\hline High Metabolic Load Distance (m) & $0.58(0.25$ to 0.71$)$ & likely moderate & $23 \%$ \\
\hline Sprint Distance $(\mathrm{m})$ & $0.22(0.09$ to 0.45$)$ & likely small & $5 \%$ \\
\hline Dynamic Stress Load (AU) & $0.80(0.61$ to 0.93$)$ & possibly very large & $64 \%$ \\
\hline Accelerations (n) & $0.52(0.21$ to 0.67$)$ & likely large & $27 \%$ \\
\hline Decelerations (n) & $0.45(0.21$ to 0.61$)$ & probably moderate & $2 \%$ \\
\hline Time Above 85\% HRmax (\%) & $-0.74(-0.25$ to -0.89$)$ & likely very large & $54 \%$ \\
\hline Average HR (bpm; HRavg) & $-0.25(0.07$ to -0.43$)$ & possibly small & $31 \%$ \\
\hline Maximal HR (bpm; HRmax) & $-0.67(-0.45$ to -0.72$)$ & possibly large & $44 \%$ \\
\hline Percentage HRmax $(\%)$ & $-0.56(-0.33$ to -0.65$)$ & possibly large & $31 \%$ \\
\hline RPE (AU; CR10) & $-0.56(-0.33$ to -0.65$)$ & possibly large & $31 \%$ \\
\hline
\end{tabular}

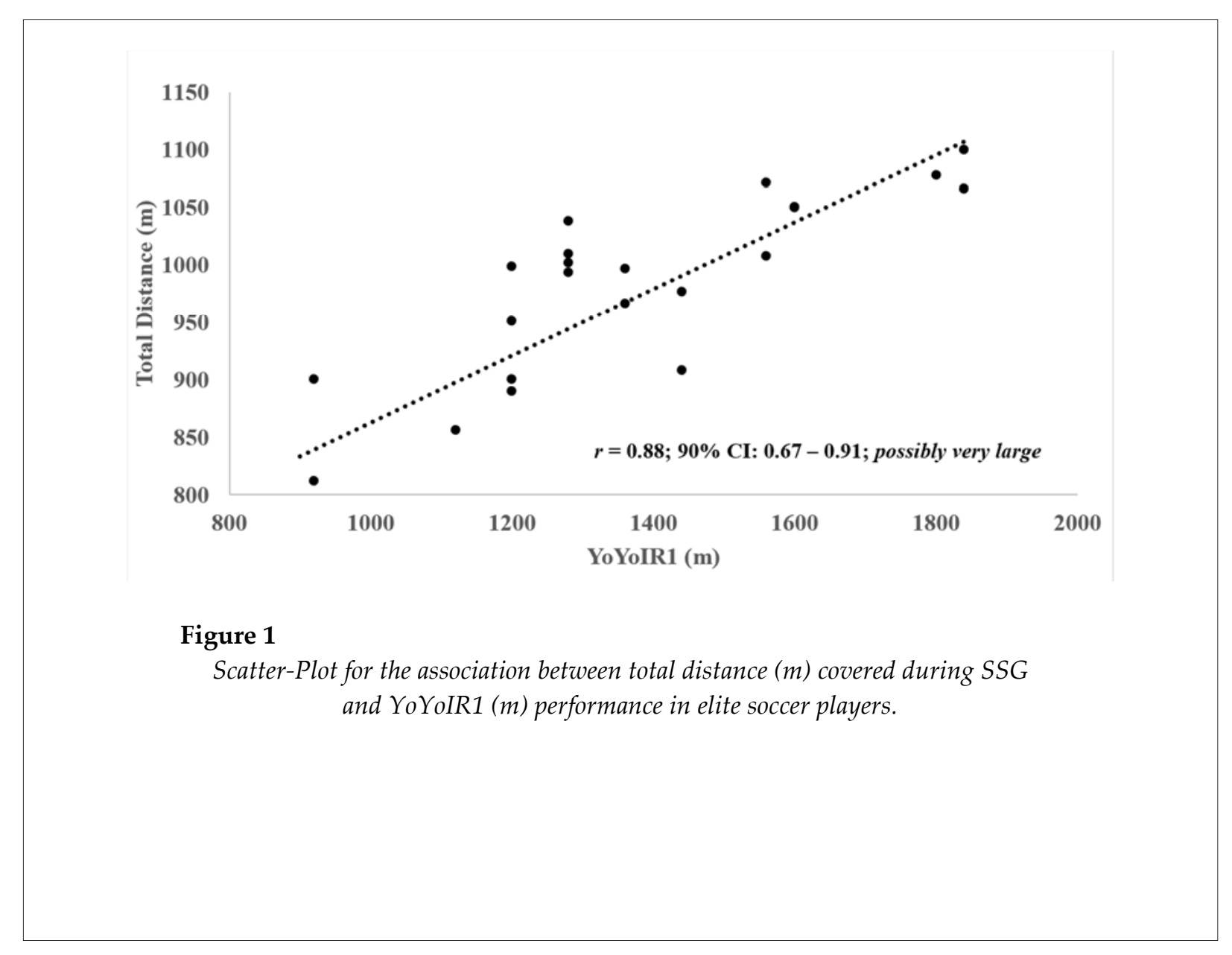




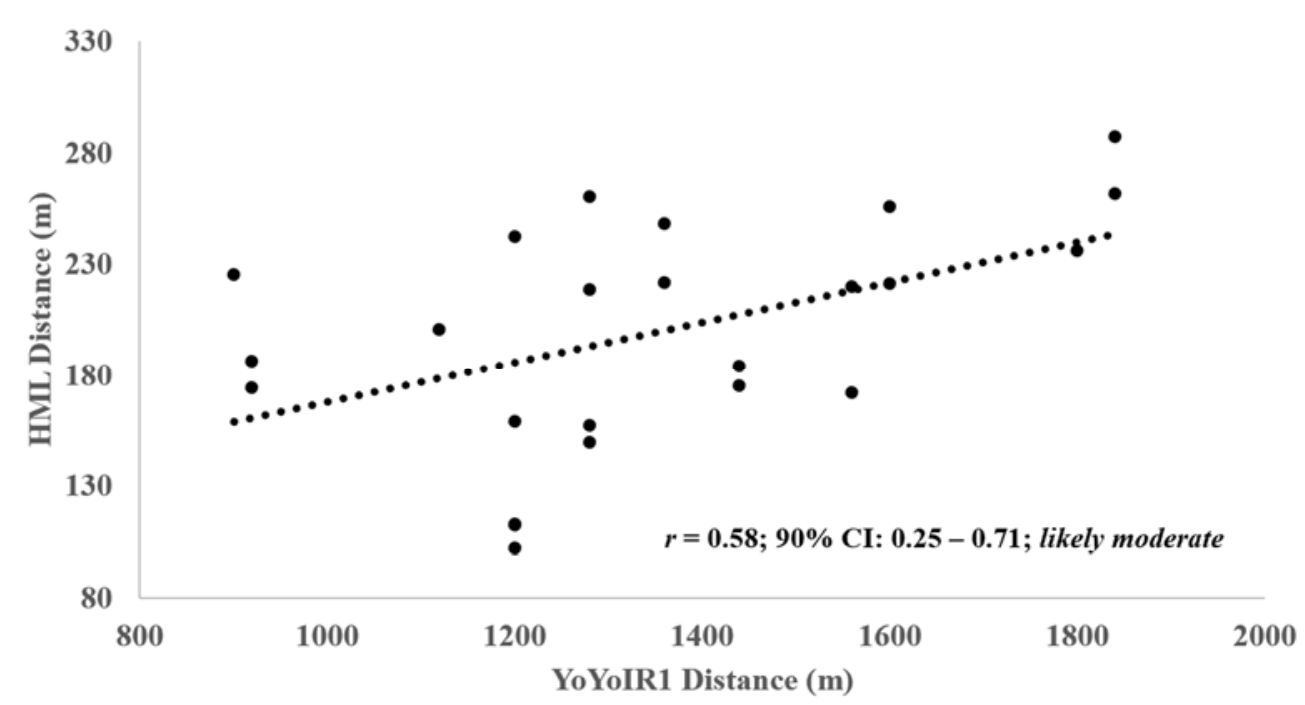

Figure 2

Scatter plot for the high metabolic power distance $\left(m ; \geq 20 \mathrm{~W} \cdot \mathrm{kg}^{-1}\right)$ covered during SSG and YoYoIR1 $(\mathrm{m})$ performance in elite soccer players.

Recently within the soccer training process, coaches have evolved towards integrated physical training with the aim to maximise training time during which players are in possession of the ball (Lacome et al., 2017). It has been shown across many investigations that SSGs can improve aerobic and anaerobic physical qualities within team sport athletes in addition to these games improving soccer-specific fitness (Dellal et al., 2012; Malone et al., 2017; Owen et al., 2012, 2014) and match winning-related factors. Within the current investigation, the 5 vs. 5 SSG protocol resulted in similar percentages of HRmax responses that were previously published in the literature within soccer cohorts. Interestingly, players spent on average $4.3 \pm 0.8 \mathrm{~min}$ above $85 \%$ of the HRmax. As such, it may be suggested that the application of these games over a training period would result in improved aerobic fitness capacities of soccer players (Malone et al., 2017; Owen et al., 2011, 2012).

Coaches are often reluctant to utilise SSGs as fitness assessments given the open loop nature of these games and potentially high variability $(\% \mathrm{CV})$ of specific running performance measures from set to set. Furthermore, coaches may understand the many factors such as pitch size, work to rest ratios, player numbers, player area, game rules, goal size, potentially impact SSG running performance. Therefore, the present investigation aimed to first assess the variability and repeatability of a 5 vs. 5 SSG protocol within an elite soccer cohort for commonly used GPS and HR variables, including the dynamic stress load (AU) and acceleration motion measures such as high metabolic loading distance (m). Interestingly, results from the current study found a low $\mathrm{CV} \%$ for global running performance measures and physiological measures. Specifically, total distance, average metabolic power and dynamic stress load presented low CV\% (>5\%). However, higher speed movements $(8.1 \%)$, accelerations $(14.1 \%)$, decelerations (16.2\%) and sprinting movements $(16.3 \%)$ were shown to have high 
variability within SSGs. These findings are in line with previous literature that has observed that high speed movement variables, acceleration and deceleration movement in addition to sprintbased variables present a high CV\% during SSGs (Hill-Haas et al., 2008; Rampinini et al., 2007; Stevens et al., 2016). During fitness assessment, most coaches are concerned with specific variables that within the current investigation presented a low $\mathrm{CV} \%$ such as total distance covered or physiological performance. Indeed, coaches will often utilise physiological data and GPS data such as total distance and time spent above the percentage of the HRmax across a week to monitor player's readiness and injury risk. Interestingly, given the low $\mathrm{CV} \%$ and high associations between the specific SSG running metrics and Yo-YoIR1 scores, it may be suggested that coaches could regress their own data during SSGs against Yo-YoIR1 data and utilise the equation to understand how each SSG completed has impacted Yo-YoIR1 running performance, given the nuances between different teams' training philosophies. As a result of the information extracted within this investigation, coaches could potentially utilise the 5 vs. 5 SSG protocol as a fitness assessment of players during specific periods of the season given the known time constraints associated with fitness assessments in soccer cohorts.

When considering SSG running and physiological performance and association of these variables with Yo-YoIR1 performance, it was observed that many variables had moderate to very large associations with Yo-YIR1 test performance. The current investigation is not the first study to relate test performance to an element of soccer specific performance. Indeed, previous studies (Buchheit et al., 2010; Casamichana et al., 2012; Castagna et al., 2010) have found similar correlations between aerobic fitness tests and match play running performance. Moreover, Manzi et al. (2014) observed moderate to large correlations $(r=0.52-0.83)$ between several aerobic fitness variables (MAS, VO2max) and distance covered in high-power categories $(>20 \mathrm{~W} \cdot \mathrm{kg}-1)$ during matches. Our findings are in line with the study by Stevens et al. (2016) who showed moderate to large associations between running performance during 6 vs. 6 small-sided games and Yo-YoIR2 running performance within professional soccer players. Specifically, it was shown that the total distance covered during 6 vs. 6 SSGs had a moderate association with Yo-YoIR2 performance $(\mathrm{r}=0.45 ; \quad 90 \% \mathrm{CI}: \quad 0.31-0.56)$; furthermore, HR measures showed similar trends within the current investigation when compared to Stevens et al. (2016) with negative associations observed between HR measures during SSGs and Yo-YoIR2 performance. However, despite the reported associations, these differed across populations from elite male to female cohorts resulting in the authors recommending caution in the thought process of the application of SSGs as a potential sole fitness assessment (Stevens et al., 2016). However, given the low CV\% for total distance and HR measures within the current and previous investigations (Stevens et al., 2016), it may be suggested that these games provide an ecologically valid measurement of aerobic fitness within elite soccer player cohorts.

The advantage of applying a 5 vs. 5 SSG protocol from a testing perspective is that this is an already frequently used methodology of training within all levels and age groups of soccer cohorts in addition to the ecological validity of SSGs being higher than traditional testing methodologies. Furthermore, the multiple use of this SSG assessment protocol can help objectively quantify changes or reductions in intermittent endurance capacities of individual players across a season. However, limitations of the current study include the fact that these data are taken amongst elite level professional players and may not be comparable across a range of playing cohorts. Additionally, not all coaches have access to the expensive equipment used within the study to provide the data capture required to assess the test. Furthermore, consideration with regard to a number of co-founding factors such as player motivation, player skill level and player pacing within SSGs may impact results of the assessment protocol. These suggested factors have previously been shown to influence locomotor performance as well as the physiological load of individual players. Therefore, given these potential cofounding factors, it may be suggested that within practice, coaches may utilise integrated training load ratios, i.e. the integration of the individualised heart rate (internal load) with distance measure (external load) during SSGs. In line with recent literature in this area, an 
additional strength of this particular investigation is the fact that this protocol may aid the identification of fitness changes and possibly counteract the potential impact of player pacing on external load variables (Akubat et al., 2014; Malone et al., 2016; Taylor et al., 2018). Given that improved aerobic fitness has been shown to reduce the odd risk of lower limb non-contact injury within soccer cohorts, and the observed associations between 5 vs. 5 SSGs and the YoYoIR1, future research may aim to best ascertain the number of exposures to 5 vs. 5 SSGs that result in improved aerobic fitness and whether this improvement is associated with reduced likelihood of players sustaining lower limb noncontact injuries. Furthermore, future research should target the use of multiple 5 vs. 5 SSG protocol assessments to view seasonal-variation in both group and individual cohorts of professional players, and even compare across various age groups or levels of professional soccer, or players of different training backgrounds or cultures. Additionally, future research may direct its interest towards the association of the 5 vs. 5 SSG assessment protocol with more laboratory physiological testing assessments.

\section{Conclusion}

To conclude, the current investigation confirms how specific measures of running and physiological performance have a low $\mathrm{CV} \%$ combined with good repeatability during the 5 vs. 5 SSG assessment protocol within elite soccer cohorts. Furthermore, these measures showed moderate to very large associations with the wellreported Yo-YoIR1 performance and as a result, promote the fact that a soccer specific 5 vs. 5 SSG assessment protocol can be utilised more regularly by coaches as a supplement to traditional aerobic fitness testing to assess the intermittent aerobic capacity of elite soccer players. Within the competitive season, coaches who find it difficult to provide time for maximal or submaximal aerobic fitness assessments due to fixture constraints within elite soccer may view this as a feasible assessment option. As such these coaches could potentially utilise these 5 vs. 5 SSGs within a typical weekly training cycle to best ascertain players' soccer specific aerobic fitness and performance capacity. The SSG protocol utilised within this investigation ultimately represents a proven reliable, time-efficient method of soccer specific assessment of intermittent aerobic fitness within elite level professional soccer.

\section{Practical Implications}

- A standardised 5 vs. 5 SSG protocol presented validity and repeatability with regard to specific metric measurements of movement within an elite soccer cohort.

- Given the low CV\% shown with specific physical and physiological variables with 5 vs. 5 SSGs, it is suggested that these games could be utilised as a time efficient method of assessing the intermittent aerobic capacities of elite soccer players.

- Consistent associations observed between distances covered in SSGs and YoYoIR1 performance suggest that 5 vs. 5 SSGs may be used as a soccer specific assessment of intermittent aerobic fitness.

- The SSG protocol utilised within this investigation ultimately represents a proven reliable, time-efficient method of soccer specific assessment of intermittent aerobic fitness within elite soccer cohorts.

- Given the fixture schedule within elite professional soccer, SSGs with a low CV\% may be placed within training sessions by coaches and used as a tool in the assessment of intermittent aerobic capacities to guide a future training prescription.

\section{Acknowledgements}

The authors would like to thank the soccer players for their participation and professionalism throughout the duration of the study; and the sport science \& medical staff. There are no funding sources and are no conflicts of interest surrounding this scientific investigation. 


\section{References}

Akenhead E, Harley J, Tweddle S. Examining the external training load of an English premier league football team with special reference to acceleration. J Strength Cond Res, 2016; 30: 2424-2432

Akubat I, Barrett S, Abt G. Integrating the internal and external training loads in soccer. Int J Sports Physiol $\mathcal{E}$ Perform, 2014; 9(3): 457-462

Banister EW. Modelling elite athletic performance. In H. Green, J. McDougall and H. Wenger (Eds.), Physiological testing of elite athletes. Champaign, IL: Human Kinetics: 403 - 424; 1991

Beato M, Bartolini D, Ghia G, Zamparo P. Accuracy of a 10-Hz GPS unit in measuring shuttle velocity performed at different speeds and distances (5-20-m). J Hum Kinet, 2016; 54(4): 15-20

Bland JM, Altman DG. Comparing methods of measurement: Why plotting difference against standard method is misleading. Lancet, 1995; $1085-1087$

Buchheit M, Mendez-Villanueva A, Simpson BM, Bourdon PC. Match running performance and fitness in youth soccer. Int J Sports Med, 2010; 31(11): 818-825

Casamichana D, Castellano J, Dellal A. Influence of different training regimes on physical and physiological demands during small-sided soccer games. J Strength Cond Res, 2012; 29(3): 690-697

Castagna C, Manzi V, Impellizzeri FM, Weston M, Barbero Alvarez JC. Relationship between endurance field tests and match performance in young soccer players. J Strength Cond Res, 2010: 24(12): 3227-3233

Dellal A, Varliette C, Owen A, Chirico EN, Pailoux V. Small-sided games versus interval training in amateur soccer players: effects on aerobic capacity and the ability to perform intermittent exercises with changes of direction. J Strength Cond Res, 2012; 26(10): 2712-2720

Djaoui L, Chamari K, Owen AL, Dellal A. Maximal Sprinting speed of elite soccer players during training and matches. J Strength Cond Res, 2017; 31(6): 1509-1517

Fleay B, Joyce, C, Banyard H, Woods C. Manipulating field dimensions during small-sided games impacts technical and physical profiles of Australian Footballers. J Strength Cond Res, 2018; 32(7): 2039-2034

Halouani J, Chtourou H, Dellal A, Chaouachi A, Chamari K. Physiological responses according to rules changes during 3 vs. 3 small-sided games in youth soccer players: stop-ball vs. small-goals rules. $J$ Sports Sci, 2014; 37-41

Halson SL. Monitoring training load to understand fatigue in athletes. Sports Med, 2014; 44(Suppl 2): 139-147

Hill-Haas S, Coutts A, Rowsell G, Dawson B. Variability of acute physiological responses and performance profiles of youth soccer players in small-sided games. J Sci Med Sport, 2008; 11(5): 487-490.

Hill-Haas SV, Dawson B, Impellizzeri FM, Coutts AJ. Physiology of small-sided games training in football: a systematic review. Sports Med, 2011; 41(3): 199-220

Hopkins WG. Precision of measurement. In: A New View of Statistics (newstats.org/precision.html); 2011

Hopkins WG. Measures of reliability in sports medicine and science. Sports Med, 2000; 30(1): 1-15

Koklu Y, Sert O, Alemdaroglu U, Arslan Y. Comparison of physiological responses and time-motion characteristics of young soccer players in small sided games: The effect of Goalkeeper. J Strength Cond Res, 2015; 29(4): 964-971

Lacome M, Simpson BM, Cholley Y, Lambert P, Buchheit M. Small-sided games in elite soccer: Does one size fit all? Int J Sports Physiol Perform, 2017; 17: 1-24

Malone S, Collins KD. The effect of game design, goal type and player numbers on physiological and physical demands of hurling specific small sided games. J Strength Cond Res, 2017; 31(6): 1493-1499

Malone S, Doran D, Akubat I, Collins K. The integration of internal and external training load metrics in Hurling. J Hum Kinet, 2016; 15 (53): 211-221

Malone S, Hughes B, Collins K. The influence of exercise to rest ratios on physical and physiological performance during hurling specific small-sided games. J Strength Cond Res, 2019; 33(1): 180-187

Malone S, Hughes B, Collins K. Are small-sided games an effective training methodology for improving fitness in hurling players? A comparative study of training methodologies. Int J Sports Sci Coaching, 2017; 12(5): 685-694

Malone S, Hughes B, Collins K. The effect of training load distribution on aerobic fitness measures in hurling players. J Strength Cond Res, 2019; 33(3): 825-830 
Malone S, Mendes B, Hughes B, Roe M, Devenney S, Collins K, Owen AL. Decrements in neuromuscular performance and increases in creatine kinase impact training outputs in elite soccer players. J Strength Cond Res, 2017 [Epub Ahead of Print]

Malone S, Owen A, Mendes B, Hughes B, Collins K, Gabbett TJ. High-speed running and sprinting as an injury risk factor in soccer: Can well-developed physical qualities reduce the risk? J Sci Med Sport, 2018; 21(3): 257-262

Mallo J, Navarro E. Physical load imposed on soccer players during small-sided training games. J Sports Med Phys Fitness, 2008; 48(2): 166-171

Manzi V, Impellizzeri FM, Castagna C. Aerobic fitness ecological validity in elite soccer players: a metabolic power approach. J Strength Cond Res, 2014; 28(4): 914-919

Owen AL, Wong DP, McKenna M, Dellal A. Heart rate responses and technical comparison between small vs. large-sided games in elite professional soccer. J Strength Cond Res, 2011; 25(8): 104-2110

Owen AL, Wong DP, Paul D, Dellal A. Effects of a Periodized Small-Sided Game Training Intervention on Physical Performance in Elite Professional Soccer. J Strength Cond Res, 2012; 26: 2748-54

Owen AL, Wong DP, Paul D, Dellal A. Physical and Technical Comparisons Between Various-Sided Games Within Professional Soccer. Int J Sports Med, 2014; 35: 286-92

Osgnach C, Poser S, Bernardini R, Rinaldo R, di Prampero PE. Energy cost and metabolic power in elite soccer: a new match analysis approach. Med Sci Sports Exer, 2010; 42: 170-178

Rampinini E, Impellizzeri FM, Castagna C, Abt G, Chamari K, Sassi A, Marcora SM. Factors influencing physiological responses to small-sided soccer games. J Sports Sci, 2007; 25(6): 659-666

Rampinini E, Impellizzeri FM, Castagna C, Coutts AJ, Wisloff U. Technical performance during soccer matches of the Italian Serie a league: effect of fatigue and competitive level. J Sci Med Sport, 2009; 12: $227-233$

Russell M, Sparkes W, Northeast J, Cook CJ, Love TD, Bracken RM, Kilduff LP. Changes in acceleration and deceleration capacity throughout professional soccer match play. J Strength Cond Res, 2016; 30: 28392844

Stevens T, De Ruiter C, Jan Beek P, Savelsbergh G. Validity and reliability of 6-a-side small-sided game locomotor performance in assessing physical fitness in football players. J Sports Sci, 2016; 34(6): 527534.

Taylor R, Sanders D, Myers T, Akubat I. The reliability and validity of integrated external and internal load ratios as measures of fitness in academy rugby union players. J Strength Cond Res, 2018 [Epub ahead of print]

\section{Corresponding author:}

\section{Dr. Adam Owen}

Inter-university Laboratory of Human Movement Biology(EA 7424), Claude Bernard Lyon 1, Lyon, France.

Email: adamowen@outlook.com 